\title{
New Single Photon Sources by Optoelectronic Tailoring of 2D Materials Using Low Energy Ion Implantation
}

Michael Hennessy ${ }^{1}$, Eoghan O'Connell ${ }^{1}$, Stefan Rost ${ }^{2}$, Manuel Auge ${ }^{3}$, Eoin Moynihan ${ }^{1}$, Minh Bui ${ }^{2}$, Hans Hofsaess $^{3}$, Beata Kardynal ${ }^{2}$ and Ursel Bangert ${ }^{1}$

${ }^{1}$ University of Limerick, Limerick, Limerick, Ireland, ${ }^{2}$ Peter Grünberg Institute, Jülich, NordrheinWestfalen, Germany, ${ }^{3}$ Georg-August-Universität, Göttingen, Niedersachsen, Germany

Monolayer thick transition metal dichalcogenides (TMDCs) with the chemical formula $\mathrm{MX}_{2}$ (M=Mo, W; $\mathrm{X}=\mathrm{S}, \mathrm{Se}$ ), constitute a new class of direct bandgap semiconductors. Their remarkable physical properties resulting from their two dimensional (2D) geometry and lattice symmetry make them an exciting platform for developing photonic devices with new functionalities [1]. Monolayer TMDCs can be easily incorporated into electrically driven devices, which in turn can be coupled to optical microcavities or photonic circuits [2]. This work constitutes a proof-of-principle study to incorporate implanted TMDCs into non-classical single photon emitting diodes [3]. The development of such devices has far-reaching implications for emerging technologies such as quantum cryptography and quantum metrology. In order to make such devices a reality, methods of material modification for these materials, such as ultra-low energy (10-25 eV) ion implantation, must be developed [4,5]. Post-growth doping [6] of TMDCs offers an expanded selection of possible dopants compared to the popular method of doping via CVD growth. The technique allows for highly pure, clean and selective substitutional incorporation of dopants [7] and is also compatible with standard semiconductor processing. Ultra-low energy ion implantation is carried out using the ADONIS mass-selected ion beam deposition system at the University of Gottingen [8].

Here we show results of ultra-low energy ion implantation of chromium into monolayer TMDCs $\left(\mathrm{MoS}_{2}\right.$, $\mathrm{MoSe}_{2}, \mathrm{WS}_{2}$ and $\mathrm{WSe}_{2}$ ). Atomic resolution high angle annular dark field (HAADF scanning transmission electron microscopy (STEM), together with core-loss electron energy loss spectroscopy (EELS) analysis, is used to identify individual dopant atoms and examine the atomic structure of the defects and dopants in the monolayers. Custom software, based on Atomap [9], has been developed to automatically identify atomic sites in experimental HAADF STEM images. Atomic positions and intensities are used to create accurate image simulations. Simulated images are refined via comparison with experimental images in an iterative process. Ab initio band structure calculations are used to analyse the suitability of individual TMDCs for electronic tailoring via ion implantation. Low loss EELS is used in conjunction with low temperature photoluminescence and Raman spectroscopy to study excitonic behavior in implanted TMDCs. Hanbury Brown and Twiss experiments were carried out to verify single photon emission from implanted sites by measuring the second-order correlation function [10].

The authors gratefully acknowledge funding from Volkswagenstiftung.

References

[1] K. Mak, C. Lee, J. Hone, J. Shan, and T. Heinz, Phys. Rev. Lett. 105, 136805 (2010).

[2] K. F. Mak and J. Shan, Nat. Photonics 10, 216 (2016).

[3] M. D. Eisaman, J. Fan, A. Migdall, Acta Med. Okayama 67, 259 (2013).

[4] K. Dolui, I. Rungger, C. Das Pemmaraju, and S. Sanvito, 1 (2013).

[5] V. P. Pham and G. Y. Yeom, Adv. Mater. 28, 9024 (2016).

[6] A. Azcatl, X. Qin, A. Prakash, C. Zhang, L. Cheng, Q. Wang, N. Lu, M. J. Kim, J. Kim, K. Cho, R. Addou, C. L. Hinkle, J. Appenzeller, and R. M. Wallace, ArXiv In Press, 1 (2016). 
[7] J. W. Mayer, 1973 Int. Electron Devices Meet. 3 (1973).

[8] M. Uhrmacher and H. Hofsäss, Nucl. Instruments Methods Phys. Res. Sect. B Beam Interact. with Mater. Atoms 240, 48 (2005).

[9] M. Nord, P. E. Vullum, I. MacLaren, T. Tybell, and R. Holmestad, Adv. Struct. Chem. Imaging 3, 9 (2017).

[10] P. Tonndorf, R. Schmidt, R. Schneider, J. Kern, M. Buscema, G. A. Steele, A. Castellanos-Gomez, H. S. J. van der Zant, S. Michaelis de Vasconcellos, and R. Bratschitsch, Optica 2, 347 (2015). 